DES 1006294

\title{
ATOMS FOR SPACE
}

\author{
David Buden
}

Published October 1990

EG\&G Idaho, Inc.

P.0. Box 1625

Idaho Falls, Idaho 83415

Prepared for the

U.S. Department of Energy

Idaho Operations office

\section{MASTER}

DISTRIBUTION OF THIS DCOUMENT IE 'JNLIMITES 


\section{ABSTRACT}

Nuclear technology offers many advantages in an expanded solar system space exploration program. These cover a range of possible applications suc as power for spacecraft, lunar and planetary surfaces, and electric propulsion; rocket propulsion for lunar and Mars vehicles; space radiation protection; water and sewage treatment; space mining; process heat; medical isotopes; and self-luminous systems. In addition, space offers opportunities to perform scientific research and develop systems that can solve problems here on Earth. These might include fusion and antimatter research, using the Moon as a source of helium-3 fusion fuel, and manufacturing perfect fusion targets. In addition, nuclear technologies can be used to reduce risk and costs of the Space Exploration Initiative. 
1. INTRODUCTION

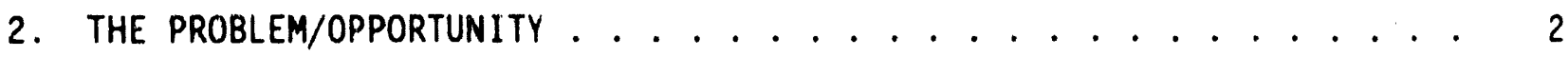

3. THE SOLUTION/THE ROLE OF NUCLEAR POWER . . . . . . . . . . . 2

4. AREAS OF NUCLEAR TECHNOLOGIES EXPERTISE RELEVANT TO AEROSPACE . . . . 3

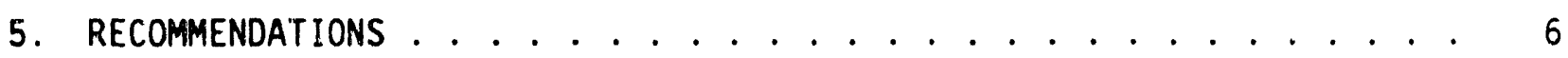

6. APPENDIX A ............................ 7

6.1 Fission Phenomena Applications............ 7

6.1 .1 Power .................. . . . 7

6.1 .2 Power Beaming ................ . . 9

6.1 .3 Process Heat . . . . . . . . . . . . . . . . . . 11

6.1 .4 Power Storage ................ . . 11

6.1 .5 Isotope Production . . . . . . . . . . . . . 12

6.1.6 Propulsion . . . . . . . . . . . . . . . . . 14

6.1.7 Large Scale Excavation And Underground Engineering . . 15

6.2 Radioisotope Phenomena Applications ........... 17

6.2.1 Electric Power . . . . . . . . . . . . . . . . . . . . 17

6.2.2 Emergency Life Support . . . . . . . . . . . . . . 18

6.2.3 Low Thrust Maneuver Units (Manned/Unmanned) . . . . 20

6.2.4 Por able Thermal Energy and Electrical Sources . . . . 20

6.2.5 Lunar Water and Sewage Treatment . . . . . . . . . . 21

6.2.6 Food Production and Preservation .......... 22

6.2.7 Self-Illumination Systems and Trail Markers . . . . 23

6.2.8 Industrial and Medical Uses ............ . 24

6.2.9 Exploration (Mineral Identification) and Processes.. 25

6.2.10 Science Applications of Radioisotope Tracers . . . 25

6.3 Science and Terrestrial Applications........... 26

6.3.1 Fusion Space Applications . . . . . . . . . . . . 26

6.3.2 H-3 Lunar Mining . . . . . . . . . . . . . . . . . 27

6.3.3 Space-Based Manufacturing of ICF Targets ....... 28

6.3.4 Matter-Antimatter Research and Applications . . . . 29

6.3.5 Advanced Accelerator Facilities .......... . 30

6.3.6 X-Ray, Ganma Ray and Cosmic Ray Astronomy . . . . . . 30

6.3.7 Neutron Astronomy . . . . . . . . . . . . 32 


\section{TABLE OF CONTENTS}

(continued)

6.4 Engineering Support Applications . . . . . . . . . . . . . 32

6.4.1 Solar Flare Warning System . . . . . . . . . . . . . 32

6.4.2 Space Radiation Protection ... . . . . . . . . . . . 33

6.4.3 Habitat Shielding .. . . . . . . . . . . . . 33

6.4.4 Radiation Interaction With Materials ........ . 34

6.4.5 Operational Management of Nuclear Systems . . . . . . 37

6.4.6 Safety/Reliability Assessment Methodology . . . . . . 37

6.4.7 Reliability In Design ............. . . 39

6.4.8 Nuclear Diagnostics . . . . . . . . . . . . . . . . . 41

6.4.9 Electromagnetic Launcher As A Space "Freight

Elevator" ...................... 43

6.4.10 Toroidal Superconducting Magnetic Energy Storage . . 44

6.4.11 A Superconducting "Storm Cellar" . . . . . . . . . . 44 


\section{ATOMS FOR SPACE}

\section{INTRODUCTION}

Nuclear energy has a major role to play in an expanded Lunar/Mars initiative program, in the conduct of more ambitious deep space missions, and in the overall establishment of humanity's extraterrestrial civilization. The ultimate role of nuclear energy will include these four basic phenomena: radioactive decay, fission, fusion, and matter-antimatter annihilation. To date, only radioactive decay phenomena (primarily in the form of plutonium-238 fueled radioisotope thermoelectric generators) has been used extensively in the performance of space missions. A number of programs have existed or currently exist in using fission reactors to generate power and for nuclear propulsion. The SP-100 program is the current major national effort to develop space fission reactors in the ten-to-hundreds of kilowatts range.

Yet, a careful review of the ambitious and challenging space activities proposed for the early portions of the next century allows one to identify numerous other aerospace applications. These include not only power and propulsion, where nuclear technology will enable certain demanding missions, but many other areas that can benefit from the application of the terrestrial nuclear technology experience. For example, space radiation protection, water and sewage treatment, space mining, and process heat are just a few of the many aerospace areas that can benefit from highly leveraged use of terrestrial nuclear technology.

Space also offers opportunities to solve problems here on Earth and to advance our scientific knowledge. It is an ideal laboratory for advanced research in such fields as fusion and matter-antimatter annihilation. It could be the source of new advanced cycle fusion fuels such as helium- 3 . Atomic research in space can help solve the U.S. and world energy problems while protecting the Earth's environment. 


\section{THE PROBLEM/OPPORTUNITY}

Nuclear technology has traditionally been applied in aerospace programs only as a last resort, i.e. when there was really no other way to do the mission. For example due to the severe environment, distance from the Sun, and long lifetime requirements, powering the Voyager spacecraft as they flew past the outer planets of our Solar system could not be handled by any other technology. Aerospace planners are however, not trained in nuclear technologies and tend to avoid "thinking nuclear". They would prefer to avoid addressing nuclear related safety issues, if at all possible.

From the perspective of the nuclear engineering community, the situation is not much better. Most nuclear engineers are trained in terrestrial systems, with little or no contact with contemporary space mission planners and understanding of space requirements. As a result of these factors, the innovative uses of nuclear technology in a variety of space-related applications has, for the most part, been ignored, overlooked or avoided.

To understand the potential benefits of nuclear technologies, one must examine the space mission requirements and recognize both the current state of nuclear technology and those technology areas in which future development could lead to major improvements. It is too early to determine which specific mission areas will be most cost effective for the application of nuclear technologies. However, even a cursory examination, indicates that many mission areas can greatly benefit from the application of existing or anticipated nuclear technologies.

\section{THE SOLUTION/THE ROLE OF NUCLEAR POWER}

A broad based program making use of nuclear technologies for aerospace missions, which we have entitled, "Atoms for Space", should be created within the U.S. Department of Energy. The program should explore all the potential uses in space of ruclear energy and nuclear phenomena to identify the technologies which are cost effective and enabling. The program should 
encompass the performance of research and development, as well as the delivery of nuclear systems which meet the goals and requirements of advanced space initiatives now under way. It should be free-thinking in its formulation and innovative in its execution. It should work closely with the National Aeronautics and Space Administration and fully coordinating its activities with other federal agencies, industry and universities.

Atoms for Space should directly support advanced space initiatives. Some of the possible mission activities are shown in Figure 1. For near-Earth missions, nuclear technology can be used to power air traffic control, communications and manufacturing platforms, provide emergency power for manned platforms, provide power for maneuvering units, movement of asteroids for mining, measurement of the natural radiation environment, provide radiation protection instrumentation, and radiation-hardened robotic systems. For the Lunar and Mars surfaces, nuclear technology can be used for base scationary, mobile, and emergency power, energy storage, process heat, nuclear thermal and electric rocket propulsion, excavation and underground engineering, water and sewage treatment and sterilization, food processing and preservation, mineral exploration, self-luminous systems, radiation protection instrumentation, radiation environmental warning systems, and habitat shielding design. Outer planet missions can make use of nuclear technology for power and propulsion.

\section{AREAS OF NUCLEAR TECHNOLOGIES EXPERTISE RELEVANT TO AEROSPACE}

The major areas of nuclear technologies with a summary description follows. In Appendix A, more detailed descriptions are provided for each area. The areas have been divided into: fission phenomena, radioisotope, science and terrestrial, and engineering support applications.

The use of fission phenomena is fundamental to the success of more demanding space missions, for example to provide power for the Lunar surface or propulsion for a one year Mars round trip. Power levels of a few kilowatts to many megawatts will be needed for surface base applications and for 


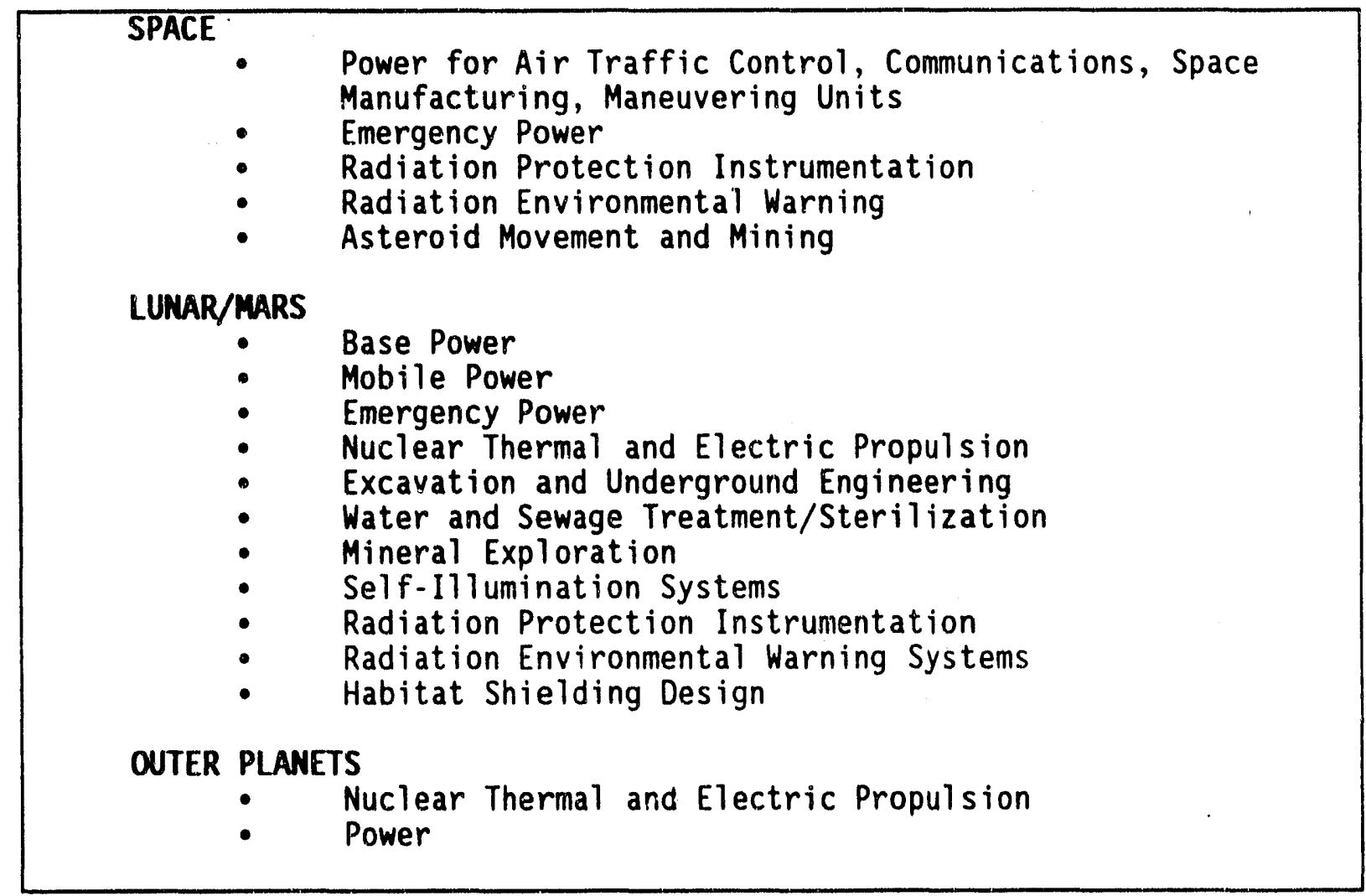

Figure 1. Nuclear Aerospace Applications

electric propulsion of cargo vehicles to Mars and deep space orbiters. Power beaming could be used to transfer energy to remote sites or for mobile vehicles. Energy storage and process heat could be used to support mining and manufacturing processes in space and on planetary surfaces. Isotope production on planetary surfaces could enable a large class of missions discussed next under radioisotope applications, such as mobile power and food preservation. Large scale excavations and underground engineering may be necessary as Lunar and Mars colonies grow, this may best be accomplished using nuclear devices.

The next calegory to be considered is radioisotope applications. Plutonium-238 in radioisotcpe thermoelectric generators has been used in over twenty space missions. There are many other useful radioisotopes, especially if they can be manufactured on the lunar surface or in space. Mobile power on planetary surfaces and maneuvering units in space can be designed to use a variety of radioisotopes. Water and sewage treatment and sterilization as 
well as food preservation are potential major applications of radioisotope technology. Nuclear technology can be used in surface and subsurface exploration for minerals. Tracer isotopes can be used to understand processes on planetary surfaces. The extremely high reliability of radioisotope heat sources makes them ideal candidates for emergency power. Self-luminous systems will be needed for emergency or remote lighting, a demonstrated radioisotope technology here on Earth. Also, portable thermal and radiation sources will be needed in the harsh environments of the Moon and Mars.

Considering science and terrestrial applications, a number of advanced nuclear technologies may be best developed in space. Space may be an ideal laboratory for fusion research, with the potential to solve energy and environmental problems here on Earth. Inertial confinement fusion targets may best be manufactured in space. The Lunar regolith and Jovian atmosphere may be mined for helium-3, a potentially important fusion fuel. Matter and antimatter research and applications may best be done in the hard vacuum of the Moon with its solid platform. Likewise advanced accelerator research may best be performed on the surface of the Moon.

Astronomy is a science that has become more and more dependent on nuclear radiation measurements. These include gamma, $x$-ray, cosmic rays and neutron measurements. The Department of Energy has a significant applicable expertise in these areas which should be applied. Understanding basic phenomena associated with the origin of the universe is an area of common interest between astronomy and the DOE community.

There are a number of engineering support applications where the expertise resides today in the nuclear technology community. Radiation protection is fundamental as we move away from the protective atmosphere of Earth. Continuous operating radiation inonitoring instruments will be needed. Methods to improve warning about natural radiation buildup driven by solar flares will be needed to protect personnel and equipment. Developing habitat shielding, mainly using indigenous materials, will be essential. The Moon is also an ideal location for a radiation biophysics laboratory to study the effects of natural radiation in an environment unprotected by an atmosphere. 
The full range of aerospace nuclear operations must be considered from the start of expanding space initiatives. This includes everything from processing radioisotopes, refueling reactors, decommissioning sites and fuel disposal. Maintenance and servicing will take on different requirements considering the value of humans and materials on planetary surfauss. We must anticipate and prepare for emergency nuclear response. The manufacturing of nuclear materials and management of processes is also needed.

There are a number of other supporting nuclear technology areas where the national expertise resides that can greatly benefit the space initiatives. These include such areas as understanding the interactions of radiation with materials, safety and reliability assessment methodology, integral design reliability methodology, nuclear diagnostics and radiation hardened robotics.

\section{RECOMMENDATIONS}

Specific recnmmendations are:

1. Establish an Atoms for Space program to ensure the full beneficial use of nuclear technologies in advanced space missions.

2. Coordinate the DOE Atoms for Space program with the cognizant users within NASA and DoD. 


\section{APPENDIX A}

Following are descriptions of various nuclear aerospace technology areas. These emphasize the possible utility of a given technology area, not the best way to perform a specific application. The intent is to illustrate the broad range of applications that nuclear technology can contribute in the performance of demanding aerospace missions and the role aerospace can contribute to solving nuclear technology issues here on Earth.

These descriptive summaries were prepared by members of the Idaho National Engineering Laboratory staff. Fission phenomena applications were prepared by: Marland Stanley, John Dearien, Jim Parks, Eric Ottewitte, and David Buden. Radioisotope phenomena application contributors included: Bruce Schnitzler, Ed Harvego, John Dearien, Eric Ottewitte, Jane Negus-deWys, David Buden, and David Woodall. Science and terrestrial applications contributors included: Thomas Dolan, David Woodal1, Jane Negus-deWys and Eric Ottewitte. Engineering support applications contributors included: Marland Stanley, David Wooda11, Bruce Schnitzler, Dennis Keiser, Vic Sturhok, John Dearien, Niall Hunt, Yale Harker, Stephen Herring and Douglas Femec.

\subsection{Fission Phenomena Applications}

\subsubsection{Power}

The requirements for power in space will be an extension of our requirements for power on earth. In fact, people in space will require an even more intense use of machines and an increased consumption of power per capita. Hundred's of kilowatts to several megawatts will be needed for transportation, habitation, communication, mining, and industry. The power levels needed will, of course, depend on the activities planned for space and extraterrestrial settlements, but these levels will go far beyond the capabilities of present power sources such as radioisotope generators and solar arrays. Table I is a summary of possible power needs of the Moon or Mars settlements at various stages in their development. 


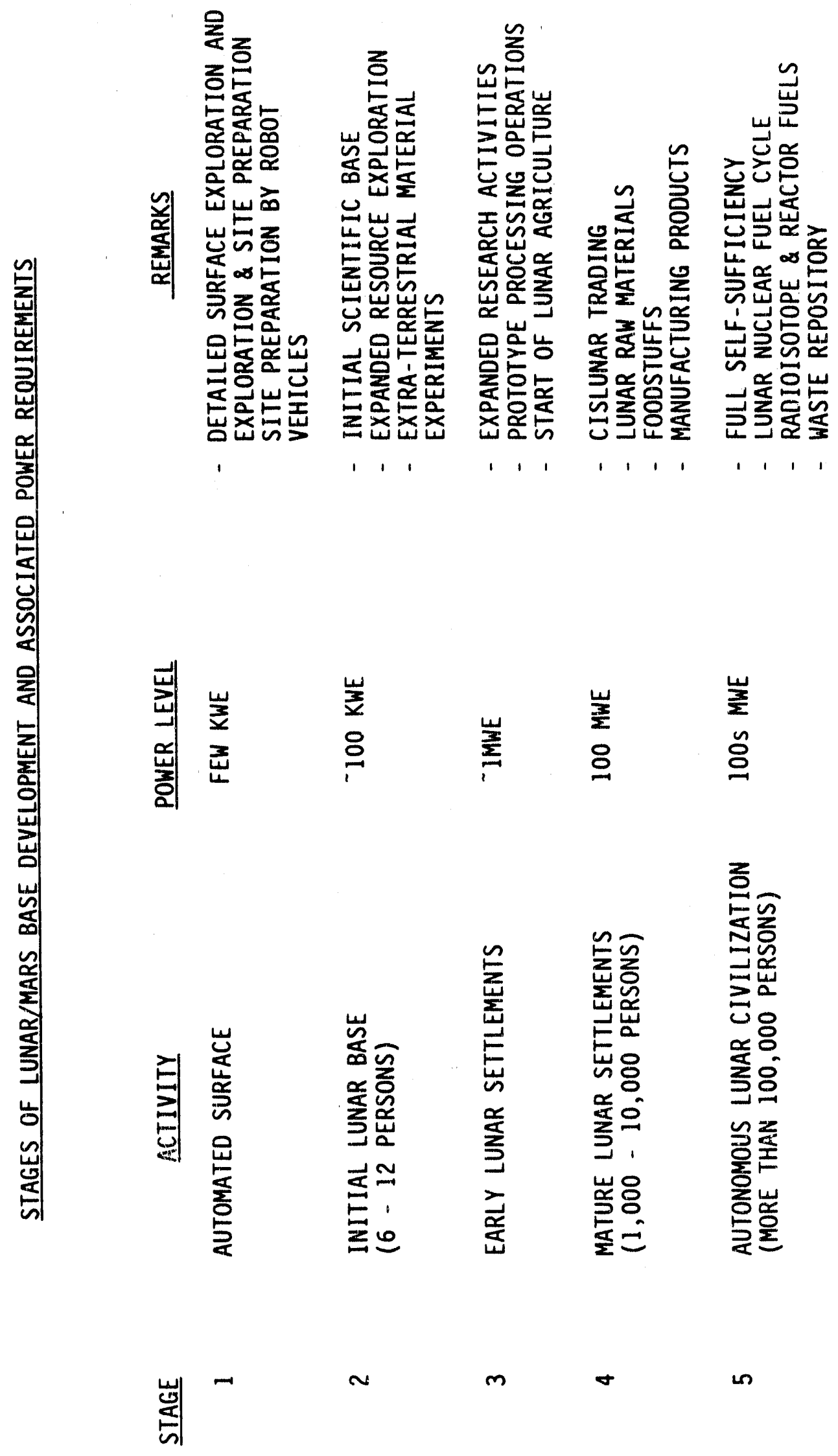


Nuclear power is essential for most lunar developments, considering the power levels needzd and the weight required for a solar/battery system. For many applications, nuclear reactors have the caparility to supply the tens of kilowatts to megawatt power levels at a weight, volume, and lifetime that puts them far beyond the capabilities of all other power sources. Also they can do sn with a closed cycle (i.e. with little or no net consumption of materials). NASA studies have shown that there are substantial savings for nuclear systems. A $500 \mathrm{~kW}$ lunar outpost nuclear power system would weigh only 12,000 $\mathrm{kg}$ compared to an advanced solar system which would weigh $370,000 \mathrm{~kg}$ : Thus, the nuclear system would save twenty-six heavy launch vehicles for each such lunar outpost.

Electric propulsion, with its very high performance relative to chemical rockets, requires the high power density source provided by nuclear fission reactors. While nuclear propulsion may not be technically enabling for propulsion, the economics and reduction of travel time available with nuclear propulsion could yery well maie it "programmatically" enabling. The use of electric propulsion for space cargo vessels, with humans following on a faster track, is a concept that is only practical with long i ife nuclear power.

Currently, the SP-100 program is developing a first generation of nuclear power sources in the tens-to-hundreds kilowatt range; this technology can be utilized for many of the initial lunar/Mars missions. Additional technology work is underway in thermionic reactors. For even higher power leve?s, the Multimegawatt Space Power Program evaluated megawatt power levels that can form the basis for solving some of the more demanding power needs.

\subsubsection{Power Beaming}

Dower beaming is a concept through which power is generated at one point and beamed, via microwave, laser, or other non-physical links, to a device which utilizes that power. The capability of nuclear sources to deveiop very high power and high power densities can be effectively utilized in conjunction with an efficient, light weight process for transmitting this power to points of use. 
There are a number of areas where the beaming of power from a generating source to a consuming entity could be advantageous. Extraterrestrial power distribution where the weight, and therefore cost, of conventional physical power links are prohibitive is one such example. Beaming the power directly from the generating station to the various users not only saves the weight and costs of physical connections, but allows for a greater flexibility in the location of facilities, up to and including the powering of mobile vehicles. The latter capability has significant imflications in extraterrestrial mining operations, which would most like?y use some sort of electrically-powered digging, loading, and hauling machinery.

Power satellites right be located around the Moon, Mars and other planetary bodies. This could simplify delivery, maintenance and operations while providing power to new bases, limited size facilities, and mobile units. Also, the orbital power system could act as a backup or emergency power grid for larger facilities.

The assembly of large vehicles, such as for manned Mars mission's, could require significant amounts of power at different assembiy locations. Also, robotic vehicles will need power for propulsion and to perform their functions. Such power requirements will be intermittent and may ta best met by a central power station.

On-orbit devices which require intermittent power include surveillance satellites, orbital transfer vehicles, devices for national defense that remain in a dormant mode for long periods of time, and material processing facilities. The savings to be realized using power beaming are (a) reduction in the number of individual power sources, (b) benefits from the economy of scale and higher power efficiencies of larger power units, and (c) extended lifetimes of space assets if on board power is a life-limiting factor.

Initial feasibility studies on power beaming have been pursued by the Strategic Defense Initiative Organization. A study by Pacific Northwest Labs, Idaho National Engineering Laboratory, and the General Electric Company explored many of the fundamental issues on the subject, such as rost, 
efficiencies, and technologies required, and concluded that beam beaming is the feasible and cost eifective solution for many applications.

\subsubsection{Process Heat.}

There are a number of space activitics which will require mainly thermal energy, primarily for human habitats and material processing needs. Process heat can be supplied directly from a reactor at temperatures in excess of 1350 $K$ and in quantities required to suppori very large habitations and material processing facilities. The energy transfer cycle can be closed, thus requiring no makeup of consumables. With the proper selection of heat transfer media, there is no transfer of radioactivity and the reactor can be designed with the proper feedback to supply power on demand.

Nuclear reactors are very efficient in the generation of heat. A liquid metal cooled reactor the size of a waste paper basket can generate several megawatts of power for many years. If one is interested in mainly the thermal output of the reactor, as opposed to the generation of electricity (at an efficiency of $6 \%-35 \%$ ), a nuclear reactor is, from a weight and volume standpoint, the most efficient device possible. Even if one of the functions of the reactor is to generate electricity, $65 \%$ to $94 \%$ of the energy generated is available for process heat.

Utilization of a nuclear reactor in the combined mode of electricity generation and the supply of process heat can result in near $100 \%$ efficiency. This highly efficient operational mode utilizes the excess heat from the electrical generation cycle as process heat for material processing or space heating in biohabitats, satisfying the heat sink requirement of the electrical generation cycle. The energy requirements of future space outposts should be designed to take advantage of such possible efficiencies.

\subsubsection{Power Storage}

Large amounts of power may be generated on the lunar or planetary surfaces. Since demand is rot likely to be constant, it may be advantageous 
to store power during low demand times for later use, or to make it more transportable for mobile or isolated facility uses. Energy storage may take several forms: nuclear, electrical, chemical, thermal or physical. The mosi appropriat! of these will depend upon the circumstances and location, but it is likely that some form of electrical or chemical storage will be used.

The storage of electricity could be accomplished by using capacitive, inductive or chemical (battery) storage. While fresent versions of battery systems tend to be somewhat massive, they will be used when they meet given requirements. Capacitors are being developed with improved storage capacity, and they may be applied for certain space application requirements. Considerable effort has gone into the development of magnei ir. storage on earth for large quantities (GJ) of energy. Again, depending upon specific requirements, this may be applied in space applications.

Electricity generated by nuclear power could be used to disassociate water into hydrogen and oxygen for use in fuel-cell-powered rover craft. Such a vehicle would generate water during use and the water in turn could be converted back into hydrogen and oxygen for reuse in the vehicle. Where water is scarce, lunar or planetary rocks could be mined for hydrogen and oxygen for use in such a vehile or for other applications such as life support systems. Other easily transportable and safely stored materials could be produced in a similar manner, where appropriate indigenous materials were available for feed stoci..

Heat from nuclear reactors could be stored in rocks and other appropriate indigenous materials for extraction at a later time, much like solar thermal storage systems being used for terrestrial applications.

\subsubsection{Isotope Production}

The use of radioisotopes in space exploration will expand significantly with each advance. Two technical issues indicate the need for isotope production in space. The high cost of launching the lense shielding material required for gamma and neutron emitters, such as C0-60 and Cf-252 is one such 
issue. Also the relatively long travel times between Earth and space facilities make it impossible to deliver short-lived isotopes, such as Mo-99 and $\mathrm{Xe}-133$. Such short-lived isotopes are used primarily in medical procedures and will become necessary in establishing on-site medical support for the space facility staff.

Radioisotopes will be a primary by-product of reactors operated for power and/or heat in space. Therefore, isotope production capability will need to be factored into the design of such reactors from the start. Target material will be initially shipped from Earth, activated at the space reactor, repackaged for use and delivered to the customer. As lunar mining operations increase, much of the target feedstock can be produced from raw materials mined on the Moon near the reactor, eliminating much of the cost of shipment from Earth. High grade steel and special alloys used in target cladding and end-product fabrication will continue to be shipped from Earth for the foreseeable future.

The design of nuclear reactors for both power production and radioisotope production must have sufficient decoupling to avoid shutdown from mechanisms associated with radioisotope production. The facility design must al so include means for transfer of samples into hot cells and for intermediate storage. Finally, it must provide for waste processing and final storage as needed. In order to produce radioisotopes in space or on the Moon, nuclear reactor need to be designed to allow for the continuous addition and removal of targets. The design must make provisions for robotically handling the radioisotopes after removal from the reactor. Extensive processing facilities will probably not be acceptable. Radioisotopes should be produced in a form that can go directly from the reactor to the application. On the other hand, environmental hazards will be limited by the biological systems present. Thus, it will be much easier to control exposure to harmful radiation than on Earth.

The initial reactor core loiding could include radioisotope irradiation target assemblies. These assemblies could be designed for ease of handing and compatibility with end-use devices. Such target assemblies will probably include heavy isotopes in the fuel regions for the production of Pu-23\& and 
Cf-252 and loadings in the poison regions for the production of $60-60$ and Ir-192. The latter isotopes can support construction activities and geological exploration and mining operations. As the facilities under construction are transformed into laboratories, machine shops, etc., the demand will expand to include the heavy radioisotopes and specialty isotopes, such as $\mathrm{Ni}-63$. As medical facilities are expanded, the demand will increase for such short-lived isotopes as Tc-99m (from Mo-99) for neutron sources for radiography and small-scale neutron activation devices. Isotopes will play an increasingly important role in space exploration and development; especially if produced in space.

\subsubsection{Propulsion}

Nuclear rockets are essential to perform Mars round trip missions in less than a year. An extensive data base exists for nuclear rockets as a result of the NERVA program of the sixties with specific impulse levels of 8.3 $\mathrm{km} / \mathrm{s}(845 \mathrm{~s})$ achieved. This is about two times the best chemical rockets can do.

Significant rocket performance gains are possible over that achieved at the termination of the NERVA program. Carbide fuel elements were operated for two hours at approximately $3200 \mathrm{~K}$ with negligible fuel loss. This technology could be used on the end of NERVA fuel elements to increase the rocket outlet temperature and performance to $9.3 \mathrm{~km} / \mathrm{s}(1000 \mathrm{~s})$.

The use of the same fuel materials in a particle bed arrangement could result in further improvement in performance. Part of this expected result is from the lower temperature drop which would exist across the fuel. Particle bed reactors can also be designed to have much lower pressure drops than the matrix type fuel elements used in NERVA. Thus, it is possible to design a very low pressure engine and take advantage of hydrogen dissociation. This becomes even more advantageous when higher temperature reactor fuels, such as refractory foams of $\mathrm{TaC} / \mathrm{ZrC} / \mathrm{UC}$, are developed to increase reactor outlet temperature to $3500 \mathrm{~K}$. One concept uses radial flow of the hydrogen to cool the moderator, followed by a fueied particle bed section to heat the hydrogen 
to close to $3000 \mathrm{~K}$, followed by the refractory foam fuel to provide even higher exit temperatures. This arrangement has the potential of $13 \mathrm{~km} / \mathrm{s}$ (1325s) specific impulse in a $22.2 \mathrm{kN}$ (5000 lbf) engine at a chamber pressure of $69 \mathrm{kPa}(10 \mathrm{psia})$, almost a $60 \%$ improvement in performance over that demonstrated in the NERVA program.

Even more significant gains are predicted for gas-core nuclear rockets, with some projections showing potential performance to $58.8 \mathrm{~km} / \mathrm{s}(6000 \mathrm{~s})$. Encouraging gas-core laboratory experiments were performed during the $60 \mathrm{~s}$ and 70s. However, proof-of-principle and demonstration tests are needed to conclusively establish the feasibility of gas-core nuclear rockets.

\subsubsection{Large Scale Excavation And Underground Engineering}

Large scale excavations and underground engineering will be major requirements for lunar and planetary development. Nuclear devices offer a light weight approach for building large caverns, excavations, surface mining and other engineering projects. Underground engineering can involve natural resource development, thermal gas storage, toxic and nuclear waste disposal caverns, recovery of geothermal heat, crushing of ore for mining, and leaching of mineral ore (under proper conditions). Surface civil engineering projects can include preparing transportation passage ways, removing overburden for mining, crushing rocks for processing, and surface preparation for structures. In addition, highly efficient seismic subsurface exploration could use nuclear explosions.

The methodologies for peaceful use of nuclear devices were explored in a program called Plowshare, which was started in 1956 and continued through the sixties. The major activity was in the stimulation of gas production in Projects Gasbuggy and Rulison. Fngineering data was obtained for nuclear explosions in granite, basalt, carbonate rocks, tuff, alluvium and salt. other ideas were evaluated, such as deep caverns for the storage of liquids or gases, the building of a new transisthmian canal connecting the Atlantic and Pacific Oceans, mining of minerals such as copper, molybdenum, nickel, and 
uranium, storage areas for radiation and hazardous waste disposal, geothermal heat recovery, and seismology exploration.

The cost of such projects on the Moon or planets would be prohibitive. using conventionai terrestrial means. On Earth, these types of activities are dependent on abundant manpower, explosives, large quantities of water, and a vast infrastructure if roads and power. Highly efficient and highly automated processes will be needed in the development of planetary resources. Nuclear devices weighing hundreds of kilograms can perform the work of hundreds of thousands of kilograms of TNT. For instance, a 19 kiloton device in an event called Blanca formed a cavity measuring $88 \mathrm{~m}$ in diameter in Nevada. With a transportation cost to the Moon of $\$ 60-100,000 / \mathrm{kg}$, the potential savings is tremendous.

One intriguing thought for the application of nuclear devices is the protection of the Earth from asteroids or large meteoroids. Suppose that radar detects a one kilometer diameter asteroid in an orbit which will collide with the Earth in about 60 days. At the time of detection the asteroid is about $320,000,000 \mathrm{~km}$ away and closing at $60 \mathrm{~km} / \mathrm{s}$. Must we just accept the pending collision that would devastate whole countries? A quick calculation indicates that a 2 MT nuclear warhead, exploded on one side of the asteroid abcut 12 days before the time of collision would be sufficient to move both the asteroid and blast debris away from the orbit of the Earth. Also, nuclear explosions might be used in a less cataclysmic sense to push small asteroids over long periods of time into orbits where they could be used as raw materials by a space station.

The major concerns in the use of nuclear devices relate to biological safety and radioactive material contamination. Operation on the Moon or other planetary body would greatly reduce the biological risk (very low population, no ground water). The degree of radioactive material contamination needs to be evaluated, especially in light of any technology developments in the last twenty years to minimize the fissile content of nuclear devices. 
Planetary engineering will be needed to shape such bodies to mankind's use. At this time it is premature to state whether nuclear devices will be a cost effective solution in the development of planetary engineering. The list of potential applications is long and will expand as lunar operations expand; the practicality of using nuclear devices needs to be assessed.

\subsection{Radiorsotope Phenomena Applications}

Presently, radioisotopes have been used as multi-hundred watt electric power sources for Earth orbit communication satellites, lunar and Mars surface scientific experiments and deep space probes. They have also been used as thermal heaters on spacecraft. These radioisotope thermoelectric generators (RTGS) primarily use $\mathrm{Pu}-238$ as the heat source for producing small amounts of electrical power.

\subsubsection{Electric Power}

Power in the hundreds of watts to many kilowatts will be needed for such applications as lunar and planetary rovers, deep space probes, special science laboratories and isolated facilities. On the lunar surface these will need to operate during a two week night cycle. Ruggedness, availability and dependability will be key attributes of these systems.

Past radioisotope thermoelectric generator (RTG) applications included the Apollo Lunar Surface Experiments Program (ALSEP) experiments, the highly successful Voyager missions, and the recently launched Galileo spacecraft. Static (thermoelectric) conversion provides high reliability and is well-suited to the relatively low power levels required for these devices. Major increases in conversion efficiency require dynamic conversion systems. Goals of the Dynamic Isotope Power System (DIPS) Program included development of technology for dynamic power systems in the 1 to $10 \mathrm{~kW}_{\mathrm{e}}$ range. Lunar and planetary surface rover power requirements typically range from 2 to $25 \mathrm{~kW}_{\mathrm{e}}$. 
A radioisotopic fuel may be selected from a large number of radioactive candidates. Important seiection criteria include availabili.y and cost, half-life, specific power, shielding requirements, and fabricability. For space applications, safety must be assured over a wide range of possible launch abort and reentry accident scenarios. Most applications to date have employed Pu-238, primarily because of the long half-life and low shielding requirements.

Lunar and planetary surface applications, such as rovers, will alter the relative importance of the above selection criteria. One intriguing possibility is on-site production of radioisotopic fuels. Nuciear power is essential for permanently manned surface outposts and the power reactor can also provide irradiation services. Very high specific power isotopes (with inherently shorter half-lives) become attractive for rover power applications. Safety concerns during Earth launch and transport to the outpost can be minimized by utilizing stable (non-radioactive) targets. Such targets can be activated at the planetary body (and reactivated) as needed.

\subsubsection{Emergency Life Support}

Emergency life support systems will be required for all phases of lunar and planetary exploration and development. Radioisotopes are potential candidate power sources for emergency life support systems because of their relatively high power densities, and their ability to operate dependably for extended periods of time without relying on solar flux or other recharging mechanisms. Radioisotope power systems can also be designed to operate reliably in extremely hostile environments with little or no maintenance or monitoring. For example, radioisotope thermoelectric generators (RTGs) using thermoelectric devices for direct conversion of radioisotope fuel decay heat to electric energy have successfully operated in a variety of hostile space mission environments for almost two decades. These include the Van Allen Radiation belts, the lunar surface, Martian dust storms, the asteroid belts, and the temperature extremes of the other planets. 
Since radioisotope power sources can be designed to produce electric power levels ranging from a few watts to several kilowatts, such power sources can be used in a variety of emergency life support applications. These include small portable backpack systems, medium sized mobile systems, and large stationary units. For small portable or mobile emergency life support systems, where size and mass are critical, RTGs can provide up to a kilowatt of electric power at an efficiency of approximately 6 to 7 percent. The major disadvantage of radioisotope power sources in such applications is the need to provide biological radiation shielding, which can add significantly to the size and mass of the life support systems. As described below, however, the required shielding for these smaller systems can be significantly reduced by the appropriate selection of radioisotopic fuels.

For large stationary life support units that require several kilowatts of electric power to operate, dynamic radioisotope power systems (DIPS) are the system of choice. They use dynamic thermal-to-electric energy conversion devices to provide up to 10 kilowatts of electric power with efficiencies of 20 to 30 percent. DIPS power conversion can use a Brayton cycle (gas working fluid) or Rankine cycle (two-phase working fluid), depending on the specific application and the availability of resources.

A variety of radioisotope fuels can be used for emergency life support power systems. For small portable or mobile systems, alpha-emitting radioisotopes will generally be preferred because of their relatively low shielding requirements and inherently high power densities. Because of its availability and relatively long half life, Plutonium-238 is the most likely alpha-emitting isotope for such application. The disadvantage of this isotope for applications other than small portable or mobile systems, is its relatively high cost. For larger stationary life support systems, and for systems that can be isolated or easily shielded, less expensive nuclear fuels such as Curium-242, Strontium-90, or Cesium-137 (among others) may be used. The decision to use one of the latter fuels in place of the more commoniy used Plutonium-238 involves a trade-off between the lower cost and the less desirable radiation and power density characteristics of the latter radioisotopic fuels. 
A final consideration in the use of radioisotopes as power sources for emergency life support systems in space is the potential to produce the radioisotopes on site at established lunar or planetary bases. This approach would considerably reduce the costs and safety concerns associated with production and delivery of these radioisotope fuels from Earth.

\subsubsection{Low Thrust Maneuver Units (Manned/Unmanned)}

Being able to move personnel anu rojotic equipment will be necessary for efficient operations in space. This will require portable, light weight power packs. One possibility for a low thrust maneuvering unit uses a radioisotope such as Sr-90 for long-duration, low thrust needs. Such a unit could be designed so that coolant and flow failures could be benign and so that the low-gamma characteristics of Sr-90 could be used.

\subsubsection{Portable Thermal Energy and Electrical Sources}

Nuclear sources, at the power level of watts to tens or hundreds of watts, provide our existing space effort with power for space probes. Such power cannot be supplied by any other means in some circumstances. Solar would not function at the distances from the sun traveled by some probes and batteries would be too heavy for the time and power level required. These applications of "portable" energy sources are well established in the space industry. As the space effort expands, there will be many more applications for which a small thermal source or electric generator are required. These could include small stations on the back side of the Moon, long life monitoring satellites around the far planets, surface stations on the inner planets, and a number of potential applications around the Earth.

There are a number of isotopes that can be used in the generation of thermal energy and several systems which can be utilized in the direct conversion of heat to electricity. The proper selection of an isotope or electrical generation system depends on the application. 


\subsubsection{Lunar Water and Sewage Treatment}

Life in a closed space station and on planetary bodies will require the processing of all sewage to allow for the reuse of water for plants and human consumption, sterilization of waste to be returned to the local biosphere, and the destruction of pathogens to prevent the spread of disease. Furthermore, before we can begin exploring the surface of Mars, or otier bodies which may have their own life-forms, it is imperative that the spacecraft be sterilized to avoid introducing earthly life-forms. Cs-137, a fission product and $\mathrm{Co}-60$, produced by neutron irradiation of natural cobalt, are gamma ray sources frequently used for sterilization.

There are no known rivers or other direct water supplies on the Moon. There are also no sewage ponds and landfills: any plans to create such would be a poor use of potentially valuable rescurces. A solution is sewage processing and recycling.

Current earth-based technologies for water and sewage treatment require abundant and cheap water and disposal sites. Manned NASA space vehicles have previously been of limited duration and have used open life support systems. While human waste is high in nutritional value, it is infected with bacteria. Thus a program of sterilization will be required to close the ecological cycle of lunar habitation.

The effects of radiation on the breakup of microscopic organisms and the sterilization of food and water have been studied for decades. Co-60, with two strong gamma rays that exceed one MeV, can provide lethal dose for organisms. The amount needed increases roughly as their size decreases. While $500 \mathrm{rad}$ ( $5 \mathrm{~Gy}$ ) is the absorbed dose which is lethal to half of the exposed human population (untreated), one million rads is required to destroy algae. In addition, radiation kills bacteria with little damage to low molecular weight compounds. The resulting sterilized products may be reused for water supply and for cattle and plant food. No nuclear activation products result from irradiation by such low energy gamma sources. 
To avoid long processing times, it is important to concentrate the radiation. This is best done by minimizing the half-life of the radioisotope used, by maximizing the radiation energy, or by physically concentrating the emitter. However, a very short half-life would require constant replacement of the source and little litilization on the treatment of waste. One might further take into account the size of the waste treatment container, the composition of the solution, and the effectiveness of the emitted radiation in depositing energy. Also, radiation alone can be used to dertroy bacteria, synergistic combination with heat has been found to be much more effective. An important potential by-product in developing waste treatment facilities for space settlements could be technology transfer to solving Earth's waste problems.

\subsubsection{Food Production and Preservation}

Far from Earth, all the "ground" rules change. Space travel and lunar residence instantly inflate the costs of food and other goods. The problem addressed here is the high cost of a food supply in space. With no soil or atmosphere, and with lunar days and nights lasting twenty-eight Earth days, farming must be substantially different than on Earth. It may follow the example of Earth hydroponics but in a gas-tight greenhouse, possibly enhanced by human-and animal-exhaled $\mathrm{CO}_{2}$. A paper at the April 1990 Idaho Academy of Science meeting showed that doubling the $\mathrm{CO}_{2}$ concentration increases plant yield by $30 \%$. Nutrients (fertilizers) will likely play different roles in space compared to that on Earth.

Once the food is grown, it will be important to use it without waste. Although controlled farming will likely not be as seasonal as on Earth, one cannot expect to engineer a continuous year round supply of each desirable food product. Also astronauts will not have access to local food supplies, since the very purpose of their effort is to explore and colonize space. Thus they must be concerned with food preservation.

The problem of food spoilage could be ameliorated somewhat by the absence of oxygen and bacterial in lunar and space environments. This can be 
stildied on Earth under simulated conditions in the laboratory. ihe use of stable and radioactive isotopes as tracers may prove useful in studying plant and animai growth under lunar simulated conditions here on Earth. Such studies could be continued on a lunar base to further optimize growth.

Radiation has been used for the conseryation of food, as a replacement for heating, canning or freezing. Principal radiation sources used are Co-60 and Cs-137 isotopes and electron accelerators. Radiation sterilization attempts to destroy all bacteria without the small amounts (ppm) of decomposition products changing the taste of the food or endangering long term health of the consumer. Radiation pasteurization (i.e. partial sterilization with lower doses) and irradiation at low temperatures cause correspondingly smalier taste changes. Extensive investigations have shown genetic risks to be extremely low for the general public and would likely be waived completely for small lunar communities. The use of radiation preservation varies by country. Foods approved for such treatment include potatoes, onions, shrimp, fried meat in plastic bags, wheat and wheat products, chicken, papaya, and strawberries. The energy requirement for radiation pasteurization of milk is $0.76 \mathrm{kWh} / \mathrm{t}$ compared to freezing, $90 \mathrm{kWh} / \mathrm{t}$, heat pasteurization, $230 \mathrm{kWh} / \mathrm{t}$, heat sterilization $300 \mathrm{kWh} / \mathrm{t}$, and drying $700 \mathrm{kWh} / \mathrm{t}$. In addition food irradiation does not require expensive packaging.

\subsubsection{Self-Illumination Systems and Trail Markers}

On the Moon, nights are long (14 Earth days). Because of lack of outside air and radiation hazards, human activity will be more confined to gas-tight, shielded central chambers. These will tend to be lighted by central power sources and their extended distribution lines. Due to its exploratory nature, however, a lunar base will always include remote areas. These should benefit from portable self-illumination systems. Landing sites for vehicles from Earth, trail markers, and markers for rendezvous vehicies are some possibie applications.

Beta-irradiation from $\mathrm{Kr}-85$ on phosphorus causes visible light. Radiokrypton light sounces have widespread applications where reliable lights 
are required. Examples are airports, railroads, and hospitals, or where sources of electricity could cause a dangerous situation, such as in coal mines and natural gas plants. However, fission product krypton contains only $4 \%$ of $\mathrm{Kr}-35$. This makes it unsuitable for high intensity lighting applications. $\mathrm{Kr}-83$ must be enriched about a factor of ten for high intensity high applications. This can presently can be done by thermal diffusion, isotopic enrichment process, but may be immensely improved by liser enrichment.

\subsubsection{Industrial and Medical Uses}

Radioisotopes are often used as medical diagnostics and in medical treatments. Co-60 sources are well suited to penetrating large pipes and chemical reactors for non-destructive examination (NDE) of materials. Suitable C $0-60$ half-life ( 5.3 years) and $100 \%$ natural abundance of target material C0-59 also contribute to its popularity as the workhorse radioisotope of industry. Cs-137, with a 30.1 year half-lite, also receives consideration on Earth.

Radioisotopes are an essential part of medical diagnostic procedures. Diagnostic methods will be needed to understand the new conditions of working and living in space. Using radionuclides in an organ makes it possible to assess the dynamic processes taking place within the organ. For example, a patient given $\mathrm{Xe}-133$ gas to breathe can be used to evaluate ventilation in the body. Radiopharmaceuticals are used in thyroid, hepatic, renal, mineral metabolism, regional blood flow, cardiac, pulmonary and gastrointestinal studies on Earth. Radiommunoassays and related procedures are very sensitive methods that can be used for measurements of hormones, enzymes, hepatitis virus, certain serum proteins, some drugs and a number of other substances.

Other uses will include such items as medical therapy and sterilization of medical products. Medical therapy uses radioiodine or Co-60 for treatment of certain types of cancer. Another major use could be in sterilization of medical products. With the limitations in facilities, sterilization by gamma rays could have major advantages. 


\subsubsection{Exploration (Mineral Identification) and Processes}

$X$-ray spectroscopy, gama ray spectroscopy, and imaging spectroscopy are well suited to lunar surface exploration for alkalic basalts, volcanic vent alteration haloes, and ores of aluminum, copper, iron, chromium, and titanium. These elements could be valuable in the exploitation of indigenous materials. Lunar soil samples recovered from both manned and unmanned lunar landings have revealed its composition consists of 42 percent oxygen, 21 percent silicon, 12 percent iron, 8 percent calcium, 7 percent aluminum, 6 percent magnesium, and 3 percent other materials, including $\mathrm{He}-3$.

Titanium can be abtained from ilmenite, indigenous to the Moon. Iron and alloyed nickel and cobalt will be easier to recover than aluminum, silicon, magnesium, or titanium. However, aluminum and other metals could be recovered from lunar rocks with processing plants that are transportable from Earth. Many volatile elements may be recoverable by processing the top $10 \mathrm{~cm}$ of scooped lunar soil. This includes the He-.? which could be used in $\mathrm{He}-3$ isotope fuel for fusion reactors.

He- 3 has been identified as one of the most important mining targets on the Moon. It is rare on Earth, but has been identified in both highlands and maria regoliths from samples returned by Apollo. Concentrations of 20 to 47 weight parts per million are recorded. The cost per metric ton delivered to Earth in the first quarter of the 21 st century is estimated at \$1 Billion, about half the cost of fuels currently used to produce electricity. He-3 is continuously implanted in the lunar soil by the solar wind. The lunar regolith is meters thick over basaltic rock.

\subsubsection{Science Applications of Radioisotope Tracers}

Radioisotopes have broad ranging application in studies of biological systems in the terrestrial environment. They would also be extremely useful tools in a lunar or other space environment for ecological and biological studies. Due to the unique, closed nature of a lunar, space or martian ecology, the availability of diagnostic tools to the space biologist could 
well determine the health and long term survival of a colony. A radioisotopeproducing nuclear reactor would be a tool to achieve this desired end.

\subsection{Science and Terrestrial Applications}

\subsubsection{Fusion Space Applications}

Several magnetic confinement fusion (MCF) and inertial confinement fusion (ICF) schemes have potential for space applications: tokamak, tandem mirror, field reversed configuration, reversed field pinch, stellarator, bumpy torus, dense $Z$ pinch, laser-driven ICF, ion beam-driven ICF, and magnetically-insulated ICF. Studies based on these concepts lead to some attractive space propulsion concepts, assuming continued success in plasma control. Deuterium and lithium fuels are abundant, and several hundred kilograms of $\mathrm{He}-3$ fuel are available on Earth. There are also proposals to mine the top meter of lunar soil, where the He-3 abundance is -12 weight parts per billion.

For space propulsion, fusion reactors could provide significant advantages over fission reactors: much more energy per fuel atom consumed (4 $\mathrm{MeV} / \mathrm{amu}$ vs. $1 \mathrm{MeV} / \mathrm{amu}$ ), higher fuel atom burnup (2 $20 \%$ vs. $5 \%$ for solid-core fission reactors), and hence, great energy yield per kilogram of fuel (chemical $\sim 10^{7} \mathrm{~J} / \mathrm{kg}$, fission (10\% burnup) $\sim 10^{13} \mathrm{~J} / \mathrm{kg}$, fusion (20\% burnup) $10^{14}$ $\mathrm{j} / \mathrm{kg}$, and matter annihilation $\left.\sim 10^{17} \mathrm{~J} / \mathrm{kg}\right)$. Also, fusion rockets provide higher thrust than ion engines and better combinations of specific impulse and thrust. Deuterium-He-3 offers the fuel safety advantages of being non-toxic, non-explosive, non-radioactive, and low neutron yield.

Short interplanetary travel times are desirable to avoid physiological damage and cancer. To achieve short trips, both high power/mass and high specific impulse are needed. In principle, fusion power plants could attain power/mass ratios $>1 \mathrm{~kW} / \mathrm{kg}$ and specific impulse values $-20,000 \mathrm{~s}$ (about fifty times chemical rockets). For an Earth-Mars mission with $33 \%$ payload, the round-trip flight would be about 80 days with a fusion power plant. In 
addition to propulsion, other potential space applications of nuclear fusion include on-board electric power, heating, neutrons for radioisotope production, and gamma rays for sterilization of instruments, wastes and food.

Development of terrestrial fusion power will require about $\$ 30 \mathrm{~B}$ investment. Present fusion experiments have achieved parameters close to those required for power reactors, but relatively low budgets ( $\$ 0.3 \mathrm{~B} /$ year) have prevented construction of full-size devices and materials testing facilities.

\subsubsection{H-3 Lunar Mining}

As mentioned above, the lunar resource of the isotope helium-3, a decay product of tritium, can provide a clean and safe source of energy on Earth for centuries. The estimated quantities are based on samples from the Apollo and Lunar programs. The source is the solar wind; the He-3 has been deposited on the lunar surface over the past four billion years and spread a few meters deep into the regolith by meteoritic bombardment. Such a resource of $10^{9} \mathrm{~kg}$ of He-3 burned with deuterium (D) would provide 2,000 years of present world energy consumption. Estimates of efficiencies of the burning of $\mathrm{He}-3$ with deuterium (D) as a fusion fuel range from 70 to $99 \%$.

He-3 placad in ari annular cell or array of cells around a tokamak fusion generator can convert moderated fusion neutrons to energetic ions by the $\mathrm{He}-3$ $(n, p)$ T reaction and thereby excite gaseous lasants mixed with the He- 3 while simultaneously breeding Tritium.

He-3 can be economical. It could be Moon mined and sold at a price comparable to oil. It has been estimated that, in the first quarter of the 21 st Century, the commercial cost of 1 metric ton of $\mathrm{He}-3$ delivered to Earth will be $\$ 1$ billion. This compares with $\$ 7 / \mathrm{bbl}$ oil at today's prices. The high energy ions released by fusion reactions can be converted to electricity with solid-state converters. He-3 is not radioactive. This offers an economic payoff for space missions. At a cost range of 100 to $\$ 500 / \mathrm{g} \mathrm{He}-3$ offers an attractive alternative to lithium as a Tritium breeding medium for 
fusion applications. Fusion research has reached a point in sime where $R \& D$ efforts to develop a $\mathrm{D} / \mathrm{He}-3$ fusion reactor can be $\mathrm{planned}$ with some confidence of success. The acquisition of this valuable fusion fuel element greatly enhances the commercial potential of the lunar Base.

Deuterium/helium-3 (D/He-3) fusion faces a more difficult physics development path, but an easier technology development path, than does deuterium/tritium $(D / T)$. A large scale operation and improved technology is required. Detailed assessment of the potential of the $\mathrm{D} / \mathrm{He}-3$ fuel cycle requires more information. Although $D / H e-3$ fusion may be best for commercial purposes, $D / T$ fusion is easier to implement in the near term.

\subsubsection{Space-Based Manufacturing of ICF Targets}

Inertial Confinement Fusion (ICF) involves the symmetric irradiation of a fusion target by high power sources of energy, either 1 asers or energetic ions. The heating of the surface of such targets results in ablation, which drives an inward implosion of the target. High temperatures and densities can be achieved when the imploding material converges, leading to significant fusion burn.

There are two symmetry requirements for ICF. The energetic source must symmetrically irradiate the target surface and the target itself must be highly symmetric. During target implosion, asymmetries in mass are amplified by Rayleigh-Taylor or fluid instabilities, leading to an imperfect implosion and low fusion energy yield.

ICF targets have been designed with multiple layers to improve the fusion yield performance. The substances used in ICF target manufacture include metals and ceramics, as well as cryogenic materials. The manufacturing process is complex, ranging from cryogenic temperatures to the melting point of metals and ceramics. The manufacture of multiple rayer ICF targets is hampered in the laboratory by the presence of gravity, which causes preferential plating of components on target surfaces. Gravity on the Earth's 
surface sets a lower limit on the symmetry which can be achieved in practice in ICF target marufacture.

Space offers the advantage fo' ICF target manufacture of negligible gravity effect (microgravity) in the manufacturing process. This offers the possibility of orders of magnitude improvement in target symmetry, leading to achievable ICF fusion energy gain and perhaps to commercial ICF fusion power.

\subsubsection{Matter-Antimatter Research and Applications}

At 2 PM. Mars Standard Time, Interplanetary Patrol Ship 3 observed an UFO some 40,000 miles away. They decided to give chase. Given today's understanding of physics and chemistry, the above chase cannot occur without the use of antihydrogen as the fuel mechanism. All other systems would require the IPS to first swing around some planet or Moon for any drastic change in direction. Only antimatter propulsion offers the specific impuise needed to turn around in space.

The value of antihydrogen can be grasped with a simple figure-of-merit: energy storage potential per unit mass. For fissionable fuel with $10 \%$ burnup, the value is $200 \mathrm{MeV} \times 0.10$ per 235 atomic mass units or roughly $0.1 \mathrm{MeV}$ per amu. For fusion systems, almost $20 \mathrm{MeV}$ can be released per fusion of a deuteron with tritium, or roughly $4 \mathrm{MeV}$ per amu before considering burnup limitations.

With antimatter the potentiai energy release by mixing an antiproton with a proton is $2 \times 939 \mathrm{MeV}$ from 2 amu, or roughly $1000 \mathrm{MeV}$ per amu. Thus antimatter propulsion offers a four order-of-magnitude increase in energy storage potential above the best presently achievable technology (fission). The major problem is learning how to store it. Obviously missing in this discussion is the weight of the power plants needed to utilize the energy sources. Nevertheless, the figures-of-merit are indicative, especially since

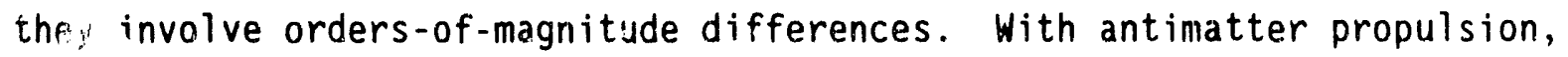
including early-generation powerplant considerations, the postulated transit time to Mars is $3-4$ weeks! 
What's the downside of all this? Antimatter propulsion has just left the level of science fiction novels (quite a few of them) and entered the more credible realm of very difficult research. Current theory on storage of antihydrogen is to use magnetically-levitated cryogenic pellets to retrieve it, a possible answer is with lasers and electromagnetic guide fields. The problems are enormous! In fact, the technology is not yet even to the embryonic state: the doors are wide open. However, the Moon is an ideal location for a full production plant; somewhat out of harm's way.

\subsubsection{Advanced Accelerator Facilities}

As the drive to explore the fundamental properties of matter pushes us to build larger and larger accelerators, we are faced with certain limitations here on Earth. The availability of the land required for a large accelerator and the isolation required for radiation shielding to protect the populace are two such limits. There are also technological and economic limitations, such as the requirement of providing high quality vacuum in a large structure.

The Moon offers two major advantages for the construction and implementation of advanced accelerators. The Moon has available high quality vacuum at no, or low, cost. In addition the Moon can provide a stable platform for an accelerator structure of arbitrary physical extent with no loss of arable or habitable land on the Earth's surface.

The availability of remote operation and maintenance, coupled with advances in data acquisition, storage and transmission, has led to a modern revolution in the conduct of large machine physics research. Research scientist.s often conduct their basic research far from the accelerator itself. Lunar habitation will be closely followed by scientific research utilization of lunar resources, such as unlimited available space and free high vacuum.

\subsubsection{X-Ray, Gamma Ray and Cosmic Ray Astronomy}

The Department of Energy has developed an outstanding capability to measure high energy particles and waves. This capability can be used to 
advantage in expanding our knowledge of astronomy. X-rays provide useful astronomical information on the behavior of complex stars, especially those which have complex interaction with other stars in multiple star systems. The $x$-ray signatures of star systems provides data on dark partners, which may be black holes or neutron stars. Our own Sun's $x$-ray image has added to the detailed knowledge of the internal workings of the Sun.

Unfortunately, $X$-rays are filtered by the upper atmosphere of the Earth, thus balloon flight and satellite instrumentation have been the only observation platforms to date. The lunar surface provides an unique, stable platform, without the interference of a lunar atmosphere. It will allow us to expand our knowledge of the universe through $x$-ray astronomy.

Gamma Ray Astronomy, like X-ray Astronomy, is hampered on the Earth's surface by atmosphere filtration. Gamina rays from astrophysical sources provide information on higher energy processes in stellar evolution. A space platform, such as a permanent space station, or the manned lunar surface, would allow for a substantial improvement in our ability to make such measurements.

Cosmic rays are extremely energetic ions which give evidence of galactic particle acceleration mechanisms, including stellar evolution processes. The detection of cosmic rays has historically been done on the Earth by measuring the shower of energetic particles produced at the surface by the collision of a cosmic ray in the upper atmosphere. Cosmic rays are an important component of the cosmic radiation background to which astronauts and non-terrestrial inhabitants will be subjected.

The study of Cosmic Rays in space is made complex by the need for new detection methodologies, since derivative showers will not exist, due to the lack of an atmosphere. Nevertheless space offers the advantage of direct observation of such particles, applying the techniques of higher energy particle physics. Synergistic new detection methods could be developed which would be ap i icable to both. 


\subsubsection{Neutron Astronomy}

The Earth's atmosphere prevents direct observation of neutrons from space. Neutrons induced by cosmic rays in the ionosphere have been measured by detectors and spectrometers borne aloft by balloons, but detailed measurements of neutrons arriving from other stars and galaxies have not been made.

Collimated detectors could scan the universe from a lunar observatory. Alternatively, the propellant tanks of a space ship may be used to shield the neutron detectors, in order to provide directional information. A narrow beam tube through the tank would permit free flow of radiation from a specified direction, determined by the angular orientation of the tank. The detector array would discriminate neutrons from other types of radiation. Long counting times would be required to obtain adequate counting statistics. Measurements of neutron flux versus direction, time variation, and energy spectra could be correlated with other types of astronomy to yield a more complete picture of the universe, and to corroborate cosmological hypotheses.

\subsection{Engineering Support Applications}

\subsubsection{Solar Flare Warning System}

The hazard of radiation in space is especially acute when there is an increase in energetic ions, for example, few MeV protons, carried by the solar wind. The flux of such particles increases periodically due to solar flare activity on the surface of the Sun which accelerated such particles. Non-terrestrial habitation demands the development of appropriate warning and detection schemes to monitor the solar mass for flare activity.

Due to the transit time of solar wind-borne particles, a warning system could be developed to alert space station and lunar inhabitants to seek radiation shelter. In addition, astronauts in transit could be provided with a protection scheme which would be deployed with enough warning. 
UTtimately, a prediction methodology could be developed based on solar observations in the visible, ultraviolet and $x$-rays. Such a predictive capability would require the continued development of a solar model and instrumentation to observe the sun. Solar observations would become a very important function of any permanent space habitation.

\subsubsection{Space Radiation Protection}

Diagnostic instrumentation will be required for the ambient radiation environment of space. This is necessary for implementation of sufficient warning for space crew members to seek shelter in the event of a significant change in the ambient space radiation environment and to measure the overall health of personnel. The instrumentation will need to encompass alpha, beta, gamma, neutron, proton, and other potential high energy particles. This type of instrumentation is a necessity to provide for appropriate levels of personnel protection during possible docking, cargo transfer, extra vehicular activities, and operation on the lunar surface.

The most common event in this category is the occurrence of solar flares, but the possible existence of other areas of concentrated radiation, such as the Van Allen belts close to Earth and high energy particles during space trips from Earth to Mars and the planets beyond.

Utilization of nuclear power or nuclear propulsion also necessitates the development of instrumentation necessary for personnel safety. Due to the vacuum of space, the mean free path of radiations encountered will be exaggerated over distances usually encountered for terrestrial radiation protection environments and consideration for particles not naturally encountered will need to be made.

\subsection{3 habitat Shielding}

Surface habitats can greatly benefit from use of lunar or martian regolith as shielaing. On the order of 10 centimeters of lunar soil appears adequate to shield against intense solar flares. However, studies are needed 
to understand the shielding values of various regolith materials and to avoid using material that could become radioactive. Regolith shielding is not "free" in that excavation and some processing, such as bagging, will be required. The required investment would be small compared to the cost of transporting traditional shielding materials from Earth.

Shielding is necessary for human exploration missions beyond low Earth orbit to protect against hostile natural radiation environments. The natural radiation hazards consist primarily of the geomagnetically trapped charged particle zones, solar wind and solar storm protons, solar flare particles, and the cosmic background radiation. Higher energy primary particles interacting in matter generate showers of secondary electrons and photons. Man-made contributions from nuclear power and propulsion sources can be minimized by design, but must also be considered.

Radiation protection for transit vehicles and surface habitats deserves special attention because of the potentially long exposure times, because of the need to protect relatively large volumes, and because of the high transportation costs per unit mass of shield. Innovative transit vehicle configurations can maximize the shielding value of the propellant and other consumables. The use of propellant for in-transit shielding is particularly attractive since the propellant mass is required anyway and the entire shield mass does not have to be braked by propulsion at the destination.

\subsubsection{Radiation Interaction With Materials}

The natural environment in space and on the Moon will expose materials to a variety of charged and highly energetic particles. The particles are similar to those the nuclear community has had to address in fission and fusion reactors and research.

Shielding against the charged particles is straight-forward. Because these particles are charged they interact readily with materials and will be absorbed in any structural material such as steel, aluminum or even plastic of about $0.5 \mathrm{~mm}$ thickness or iess. The problem with these particles is, that in 
a high flux of particles such as in the solar ind, the particles absorbed by a metal tend to cause surface embrittlement of the metal. Thus, metal exteriors will eventually become brittle, crack and crumble, unless they are annealed.

Neutrons will pass through materials and interact by scattering for the majority of the neutrons, and by absorption for a fraction of the neutrons. If a neutron is absorbed by a material, an atomic nucleus of the absorbing material is transmuted and becomes an isotope of the original nuclei with an atomic mass increased by one. The transmuted nucleus will, in all likelihood, also be radioactive. Thus, shielding of the robotics against neutrons won't be a problem unless it is desired to prevent nuclear transmutation of a robotics material. It is anticipated that some solid state electronics may be effected by this process. These kinds of components may be shielded by encasing the component in a material containing a fairly high amount of hydrogen, such as "lucite", and lining the enclosure with a material containing boron, such as "Boraxo" soap. The higher energy neutrons will then scatter and slow to thermal energy in the lucite and these thermal neutrons will the be absorbed in the boron.

The electromagnetic radiation that needs to be shielded against is primarily infrared, visible light and ultraviolet radiation. These spectra of electromagnetic radiation are easily absorbed by the exterior surfaces of the robotics and will cause heating of these surfaces. To shield against the effects of this heating all that needs to be done is to provide a reflective surface on the exterior.

A good practical shield that would protect the robot from both the solar wind particles and the thermal electromagnetic radiation would be a laose fitting "Milar" suit coated with a shiny layer of reflective aluminum. Hence, when the Milar suit deteriorated from bombardment by the solar wind particles it could be quite inexpensively and easily changed on site by lunar personnel or other robots. 
Any power source based on fission or fusion produces an abundance of high energy neutrons (assuming the fission reactor is fast). These neutrons can interact with materials in a variety of ways that can result in atom displacement, void development, and formation of new atomic species through a variety of nuclear reactions. The type and degree of charge is dependent on a number of factors including the material itself with its impurities and microstructure, neutron energy, neutron dose, and temperature. Perhaps the most significant change is embrittlement due to atom displacement. Irradiation can negatively influence the mechanical and physical properties of materials and lead to premature failure. These influences must be considered in the design, material selection, testing, and operation. Candidate materials must be tested and qualified over the range of iemperatures, fluence, and dose rate ( $f \backslash u x$ ) expected in operation.

For terrestrial power reactors where pressure vessel integrity is a prime concern, a series of capsules containing mechanical test specimens of the identical material used to construct the pressure vessel (including weldments) are placed inside the vessel in a position where the specimens will be exposed to the same neutron fluence as the vessel wall. Capsules are periodically removed and the specimens tested to determine mechanical property changes and shifts in the ductile - brittle transition temperature. Continued operation of the reactor depends upon the results of these tests.

It is probably not reasonable to assume that similar testing can be performed in space. Nondestructive techniques (NDE) that continuously or periodically monitor the properties of interest are needed. For mechanical property evaluation it may be possible to correlate some readily measurable physical properties such as electrical resistivity or ultrasonic transmission characteristics to damage.

Noncontacting NDE techniques, in which the sensors are some distance away from the object being inspected, are currently being developed for process control applications. These same techniques may be adaptable to remote inspection of components, where severe environments would preclude use of normal inspection techniques. In addition to radiation damage, these NDE 
techniques could also be used to monitor other types of degradation, such as corrosion or defect formation resulting from stress and fatigue.

\subsubsection{Operational Management of Nuclear Systems}

Operation of a power generation system in space requires that stringent procedures be developed for all aspects of the cycle, from manufacture and launch of the power system to ultimate disposal of a spent reactor. Reactors can be manufactured and kept in a very benign mode prior to their first use (as opposed to the potential energy so easily releasable from chemical reactants). Once they are turned on, however, they must be operated, maintained, and disposed of with a certain amount of care. Shielding must be provided not only for humans but for certain hardware items during operation of the unit. Maintenance of such a valuable asset is imperative, and procedures must exist in order for this to be done safely and efficientiy. At the end of life of a reactor, or at least the core, procedures must exist through which the long lived radioactive inventory of the core can be disposed of in a manner consistent with human safety and potential impact on the environment.

The basic requirements for operation of a nuclear power system are similar whether the system is in ortit or on an extraterrestrial body. In-orbit operations have some specific requirements due to tile possibility for reentry to the Earth's atmosphere; specific requirements must be addressed to the satisfaction of society. Recent problems with returning Russian satellites have emphasized the importance of this issue.

\subsubsection{Safety/Reliability Assessment Methodology}

Safety in space activities is a critical issue. Problems may originate with a launch vehicle accident, an unplanned re-entry of a space vehicle, or from irradiation of the space traveler or space inhabitant. In the latter case, safety issues will be much broader than those of accidents originating within man made processes, but, must also include the potential for inadvertent chronic and acute exposures to hazardous levels of radiation 
engendered by natural processes in the extra-terrestrial environment. The techniques for assessing the risk from each hazard can isse methodology :imilar to that developed to assess safety in the nuclear industry.

The risk assessment process generally includes each of the elements outlined below:

The hazards of concern.

The targets of concern, which could be the general public, the astronauts, and both the terrestrial and extra-terrestrial environments.

The barriers which provide the confinement of the hazard and prevent exposure of the target to the hazard. These barriers can be both physical and administrative.

The effects of target exposure to the hazard.

This information is used in the development of the complete set of scenarios, each of which details the sequence of events from an accident initiation, its propagation to an identified target damage state, and the resultant effects on the target. The frequency of occurrence for each scenario is assessed from information which:

details the frequency or probability of the accident initiators (frequency of barrier challenge).

quantitatively defines the reliability of the barriers (probability of barrier failure following a challenge).

the degree of exposure of the target following a barrier failure (hazard transport mechanisms and hazard magnitude at the target).

the damage levels incurred by the target following its exposure to the hazard and their resultant effects (consequences).

The combination of quantitative estimates for each of the contributors to risk identified above provides an estimate of the expected consequences from a specific scenario which, when combined with the contributions from all other scenarios, provides an estimate of the total expected consequences or the "risk" to the target. The consequences are typically measured in health 
effects, if the target is human, or in economic terms if the consequences result in damage to the environment.

To ensure the attainment of the expected very low levels of acceptable risk for the general public and participants in future space exploration activities, the systems and hardware which will comprise the barrier, must have very high levels of reliability. This high reliability is typically achieved by using both redundant and/or diverse hardware which provides multiple success paths. The problem tlien becomes one of ensuring their independence, because there is always some degree of coupling between their failure mechanisms, either through the operating environment (events external to the hardware) or through functional and spatial dependencies. A functional dependency could be represented by a support system which is needed to power, control, cool, or lubricate physically redundant trains of hardware and a spatial dependency could be represented by two trains of fully redundant hardware sharing a particular location in which cooling or motive fluid could be released by one (causing failure) and lead to an explosion which fails the other. A fault on one electrical bus which is not physically and electrically isolated from another, may lead to a similar concurrent failure on the second bus and result in total failure.

As the reliability of individual systems and subsystems increases, the contribution from common cause failures becomes more important and at some point dominates the overall reliability. Including their effects in the reliability models is difficult and requires that sophisticated fully integrated models are developed and solved to find the failure combinations which lead to mission failure. It is through this typically deductive and integratad modelling process that insidious failure events can be identified and corrected actions taken.

\subsubsection{Reliability In Design}

Competing needs for proposed multi-year space missions of the future, (low cost, low launch weight, and very high levels of reliability) necessitate the exploitation of new reliability engineering approaches and processes. 
These approaches must be initiated during conceptual development of the hardware and remain the primary optimization tool throughout the design process if the hardware developer is to capitalize on the cost savings which result from hardware systems integration and optimization while the design is "still on paper". The needed tools and approaches to achieve "integral design reliability" exist or are under davelopment and can be applied immediately. There are several distinct methodological approaches to achieve design optimization. Their full integration into the design process represents the culmination of seven years of development, which was first presented in early 1990 during a three day workshop held in Idaho Falls.

The distinct analytical elements, which complement existing deterministic and mechanistic techniques used in design develcpment and assure the consideration of reliability and maintainability issues from the outset involve:

Derivation of the functional objectives from mission objectives to provide the design basis for the hardware, and its description in the form of a hierarchal goal tree.

Development of logic models whose solution provides both qualitative reliability characteristics for hardware systems and quantitative assessments of the system or mission success and failure probabilities.

Prediction of failure probabilities for individual components which are based on the likelihood that material loading or stresses exceed the material strength.

There are many advantages to this approach, but the most important are:

The approach does not preclude any options "a priori" and forces the designer to fully evaluate all possible solutions. In addition, the thought process involved tends to focus a designer's attention on the precise problems of concern and can often allow the identification of unique and innovative solutions.

The complete goal tree model for the hardware provides a detailed description of its design basis, thus ensuring that as future design changes are implemented in the optimization process, the full effects of changes are recognized before they are implemented. 
Clear identification of any design "choke points" or potential mission ending single point failures.

Prediction of the system reliability or unreliability when the individual component reliability characteristics are known.

A tool which can be used to determine the "worth" of a change to the design. This worth is measured as a change in system reliability which is predicted as the reliability, redundancy or diversity of hardware is changed. This is the primary tool for system optimization.

A detailed description of how and where failures initiate and propagate, and allow a designer to build in defenses against them.

An understanding of the important failure mechanisms which can be used to advantage in a prototypical testing program. The designer is able to understand the information which would be emitted as various failure modes become active, and can use this to both identify the information which should be gathered during a test, and to ensure that provision is made in the design for the collection of the requisite data.

\subsubsection{Nuclear Diagnostics}

Nuclear diagnostics refers to those techniques which rely on nuclear radiation as the means to assay materials or examine structural components. There are many techniques which fall under this classification, however the two most popular are neutron activation analysis (NAA) and neutron radiography. These two techniques and others are adaptable to space environments and can be operated remotely. They can be used in manned space stations, unmanned space probes, or lunar settlements.

The neutron activation analysis (NAA) technique utilizes the unique properties of the neutron to probe and activate sample materials. The radiation signatures from the activation products are detected and analyzed to produce elemental composition of the sample. This technique is non-destructive and requires minimal sample preparation. Neutrons of all energies are used to perform NAA, however, thermal neutron activation is the most widely used. The sources for these neutrons are primarily nuclear reactors, however accelerator sources are used and lend themselves to remote 
applications such as space probes. Possibly the most applicable variation of the NAA technique is the prompt gamma activation analysis. This approach relies on the gamma radiation signature emitted immediately following the capture of the neutron by the nucleus. For example, probes utilizing this method have been developed for down-hole well logging in oil and mineral exploration. It was also planned in the NASA APOLLO lunar ianding missions that a combined neutron gamma experiment would be attached to the lunar rover vehicle as a means of assaying the elemental composition of the luriar 1andscape. (Unfortunately, the APOLLO mission was terminated before this experiment was scheduled to $f(y)$. Nevertheless, NASA utilized the general aspects of this methodology in analyzing the lunar samples brought back to the Earth. It is envisioned that neutron activation analysis will play a major role in future space missions as it did in the lunar landing missions.

The neutron radiography technique is similar to and is used in a complementary role to $x$-ray radiography. Relying on the interaction characteristics of the neutron, it produces a transmission image of the object. Radiography is particularly useful in examining structures involving low-atomic-number elements such as hydrogen, carbon, aluminum, etc. Examples of applications are: examination of explosives and examination of composite materials such as air foil structures. Historically the technique relied on nuclear reactors for the source of neutrons, which limits the use of the technique to examinations of materials which can be moved to the neutron source facilities. However, there has been considerable effort in developing the portable systems. Such developments would be critical to the application of neutron radiography in space programs. These efforts have focused on developing portable neutron sources of sufficient intensity and on developing high resolution real-time images. With respect to the portable sources, compact accelerator development is the major effort. Possible alternatives to accelerator sources are natural radioactive sources such as $c f-252$, however the shielding requirements for natural sources restrict their portability. The recent commercialization of the radio frequency quadruple (RFQ) compact accelerator is a prime example of how far accelerator source development has progressed. It is now possible to produce a neutron source of $10^{\text {"1 }}$ neutrons per second with a power requirement of only $15 \mathrm{kVA}$. More intense sources are 
also possible, but they would require correspondingly higher power requirements.

Originally neutron radiograph images were produced directly by exposing photographic film to the neutron beam or indirectly by exposing photographic film to a metal foil which had been activated in the neutron beam. Both processes required intense neutron sources and photographic film processing capabilities. These requirements would be prohibitive when using neutron radiography in space applications. Real-time imaging has distinct advantages, because the images are produced using current video technology and can be stored electronically. In addition, real-time imagining would require only moderate intensity neutron sources, which can be produced by the new generation of compact accelerators.

\subsubsection{Electromagnetic Launcher As A Space "Freight Elevator"}

Several space projects require large quantities of propellant, food, water, oxygen, shielding and structural materials. One attractive means for placing that material in Earth orbit is the Electromagnetic Launcher (EML). Research on electromagnetic guns/launcher is occurring within DOE laboratories. The launcher, using energy stored in a magnetic field, accelerates a one ton payload to approximately $10 \mathrm{~km} / \mathrm{s}$ with an acceleration of 1000-2000 $\mathrm{g}^{\prime} \mathrm{s}$. The payload penetrates the atmosphere, ablating and/or absorbing the aerodynamic heating.

One particular application for the EML would be to launch propellant for the nuclear thermal rocket. Since a nuclear thermal orbiting transfer vehicle is limited more by propellant than by the energy in the nuclear fuel, the orbiting propellant tank could service several missions.

Shielding against natural cosmic radiation will be required on any extensive space missions. Storm cellars will need to be provided against cosmic radiation burst. A formable material, such as borated heavy concrete, could be launched into orbit by the EML. Also, shield materials for 
protection against nuclear reactor radiation can be delivered in the same manner.

Long-duration missions will also require adequate quantities of food and water, both of which can withstand the acceleration of an EML. And finally, structural materials for construction of orbiting or lunar colonies could be launched. In all these roles, the EML could be integrated into mission as a "freight elevator" for the needed materials.

\subsubsection{Toroidal Superconducting Magnetic Energy Storage}

A superconducting magnet is an attractive energy storage device when the discharge time is 1 - 10 seconds. A disadvantage with presently proposed solenoidal energy storage magnets is the strong fringe field, which funnels charged particles into the bore of the magnet, affects humans nearby, and induces eddy currents in passing conductors. The fringe field can be avoided by arranging the magnets in a toroidal configuration. The magnetic field lines are closed within the bore of the magnet and the very small external field is caused only by the inhomogeneity of the torus.

Toroidal energy storage magnets could be used to power electromagnetic launchers, high power manufacturing processes, pulsed radio transmitters and other equipment needing intense 1 - 10 s bursts of electrical power. If the cross-section of the magnets is D-shaped, rather than circular, the windings are loaded only in tension, minimizing the amount of structural material necessary. The magnets can be built in modular sectors and assembled at their final destination. Either conventional low temperature superconductors or the ceramic higher temperature superconductors could be used. A coolant reservoir, holding either liquid $\mathrm{He}$ or $\mathrm{N}_{2}$, would be located within the toroidal bore of the magnet.

\subsubsection{A Superconducting "Storm Cellar"}

During long-duration space missions, unanticipated solar flares or other cosmic radiation will require shielding of the crew. Concentric solenoidal 
superconducting magnets could be used to produce a magnetic field with which the charged particles are guided around the spacecraft without collision. While avoiding collisions of the charged particles with materials in the spacecraft, showers of gamma rays, neutrons and other neutral particles, which require more massive shielding, are not avoided.

The spacecraft would be located in a "magnetic island" within a large magnetic field and charged particles would be guided through an annulus surrounding the spacecraft. 

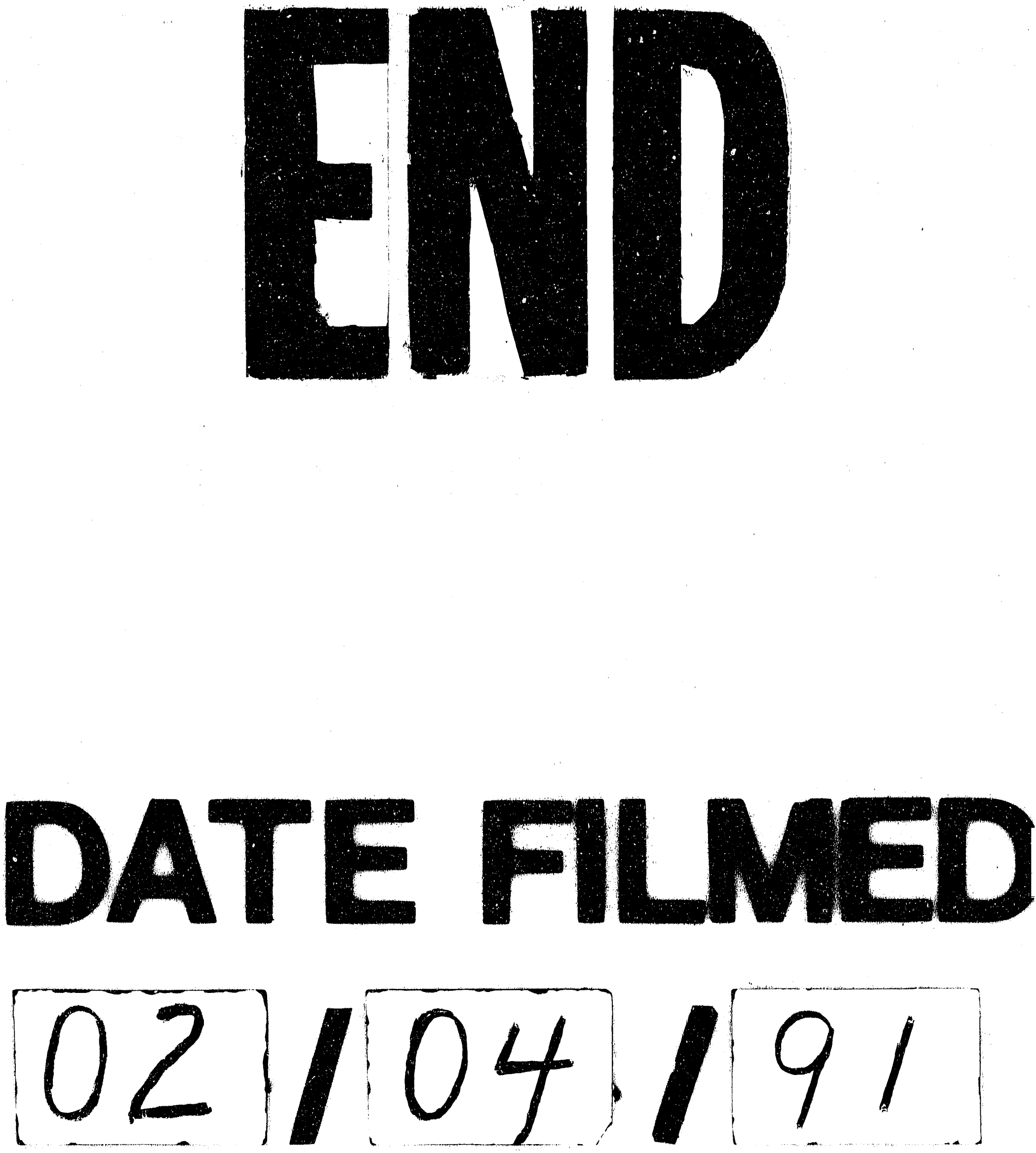
